\title{
Defining new criteria for selection of cell-based intestinal models using publicly available databases
}

\author{
Jon Christensen ${ }^{1 \dagger}$, Sara El-Gebali ${ }^{2 \dagger}$, Manuela Natoli ${ }^{3 \dagger}$, Thierry Sengstag ${ }^{4}$, Mauro Delorenzi ${ }^{5,6}$, Susanne Bentz $^{2,7}$, \\ Hanifa Bouzourene ${ }^{8}$, Martin Rumbo ${ }^{9}$, Armando Felsani ${ }^{3}$, Sanna Siissalo ${ }^{10}$, Jouni Hirvonen ${ }^{10}$, Maya R Vila ${ }^{11}$, \\ Piercarlo Saletti ${ }^{12}$, Michel Aguet ${ }^{6,13}$ and Pascale Anderle $2,5,6,7,14^{*}$
}

\begin{abstract}
Background: The criteria for choosing relevant cell lines among a vast panel of available intestinal-derived lines exhibiting a wide range of functional properties are still ill-defined. The objective of this study was, therefore, to establish objective criteria for choosing relevant cell lines to assess their appropriateness as tumor models as well as for drug absorption studies.

Results: We made use of publicly available expression signatures and cell based functional assays to delineate differences between various intestinal colon carcinoma cell lines and normal intestinal epithelium. We have compared a panel of intestinal cell lines with patient-derived normal and tumor epithelium and classified them according to traits relating to oncogenic pathway activity, epithelial-mesenchymal transition (EMT) and stemness, migratory properties, proliferative activity, transporter expression profiles and chemosensitivity. For example, SW480 represent an EMT-high, migratory phenotype and scored highest in terms of signatures associated to worse overall survival and higher risk of recurrence based on patient derived databases. On the other hand, differentiated HT29 and T84 cells showed gene expression patterns closest to tumor bulk derived cells. Regarding drug absorption, we confirmed that differentiated Caco-2 cells are the model of choice for active uptake studies in the small intestine. Regarding chemosensitivity we were unable to confirm a recently proposed association of chemo-resistance with EMT traits. However, a novel signature was identified through mining of NCI60 Gl50 values that allowed to rank the panel of intestinal cell lines according to their drug responsiveness to commonly used chemotherapeutics.

Conclusions: This study presents a straightforward strategy to exploit publicly available gene expression data to guide the choice of cell-based models. While this approach does not overcome the major limitations of such models, introducing a rank order of selected features may allow selecting model cell lines that are more adapted and pertinent to the addressed biological question.
\end{abstract}

Keywords: Cell lines, Genomic profiling, Malignant traits, Epithelial-mesenchymal transition, Intestine, Colon cancer, Chemosensitivity

\footnotetext{
* Correspondence: pascale.anderle@ibmm.unibe.ch

${ }^{\dagger}$ Equal contributors

${ }^{2}$ Institute of Biochemistry and Molecular Medicine, University of Berne, Berne, Switzerland

${ }^{5}$ Swiss Institute of Bioinformatics, Lausanne, Switzerland

Full list of author information is available at the end of the article
}

\section{Ciomed Central}

(c) 2012 Christensen et al.; licensee BioMed Central Ltd. This is an Open Access article distributed under the terms of the Creative Commons Attribution License (http://creativecommons.org/licenses/by/2.0), which permits unrestricted use, distribution, and reproduction in any medium, provided the original work is properly cited. 


\section{Background}

A wide panel of intestinal cell lines is being used to study the biology of the intestine. All of these cell lines are either directly derived from primary colo-rectal cancers (CRCs) of different clinical stages and differentiation grades or from metastatic sites originated from a colon tumor. The major oncogenic pathways in colon cancer include loss of function mutations in APC, TP53 and SMAD4 (approximately $80-85 \%$ of sporadic tumors), or DNA mismatch repair genes, and activating mutations in beta-catenin [1]. As a consequence, the Wnt pathway is activated in most tumors and derived CRC cell lines, albeit to a different extent, depending on the genetic lesions [2-5]. The morphology, expression of differentiation markers, migratory characteristics and their potential to form metastases differ vastly between the cell lines [5-8].

While cancer drug discovery has mainly focused on targeting tumor cell proliferation, the outcome of a cancer depends largely on tumor invasion and dissemination [9]. Recent advances in understanding underlying mechanisms in cancer biology including cancer stem cell (CSC) properties and epithelial-mesenchymal transition (EMT) and their relation to drug susceptibility require that relevant traits are considered for choosing appropriate cell-based models. It is believed that at the invasive front, the tumor cells undergo EMT resulting in increased migratory capacity. Furthermore, EMT has recently been linked in breast cancer to stem cell like properties [10] as well as resistance to chemotherapy in different tumor types including CRC [11-14].

The variety of available human cancer cell lines reflects the genomic heterogeneity across the human cancer population at least in part and has therefore regained attention notably to predict responsiveness of anticancer drugs $[15,16]$. Starting with the landmark paper by Scherf et al. [17] numerous studies followed that aimed at linking drug response in terms of growth inhibition with gene expression signatures, some specifically focusing on colon cancer (e.g. [18-26]). To our knowledge, no study has specifically focused on linking expression of malignant traits (i.e. EMT, WNT activity, stemness signatures) in colon cancer cell lines to response to therapy.

In this study we compare for the first time gene expression signatures relating to a wide panel of commonly known intestinal cell lines, primary cell cultures of human cancer-associated fibroblasts and laserdissected human colonocytes, small intestinal enterocytes and tumor cells. We delineate selection criteria for CRC derived cell lines based on genomic expression patterns related to clinical parameters, migratory capacities and proliferative activities. While some cell lines are mainly being used to study mechanisms associated with tumor biology others serve as models for normal enterocytes studying drug absorption. Thus, we used the same strategy to assess the validity of models for oral drug absorption.

\section{Results}

Most dominant gene expression differences as revealed by principal component analysis (PCA) were observed between normal epithelium and all tumor-derived cell lines, and cells with epithelial versus mesenchymal properties.

PCA was performed to assess the major gene expression differences between microdissected normal and tumor epithelium, primary cancer-associated fibroblast (CAF) cultures and the various CRC cell lines. PCA involves a mathematical procedure that transforms a number of possibly correlated variables into a smaller number of uncorrelated variables called principal components. Hence, the first principal component accounts for as much of the variability in the data as possible and each succeeding component accounts for as much of the remaining variability as possible. Two breast cancer cell lines with known pronounced (MB231) or weak (MCF7) EMT traits [27] were included into the comparison. The first component was defined mainly by the difference between small intestinal enterocytes and all CRC cell lines grown to subconfluency, while the second component was delineated by the difference between CAFs and epithelial cells (Figure 1). T84, HT29 and Caco-2 cells grown to confluency for 3 weeks seemed to be most similar to the small intestinal enterocytes, and also to laser-dissected tumor cells, while SW480 and MB231 proved closest to CAFs.

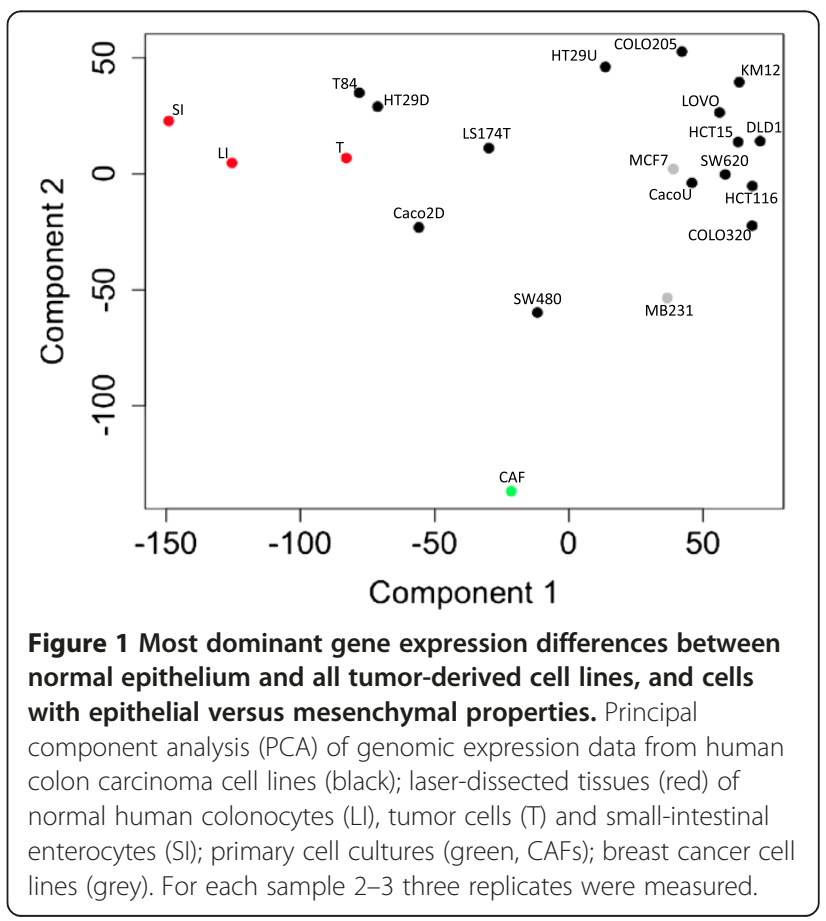




\section{Selection of relevant cell lines to study tumor biology based on expression of signatures related to malignant traits}

Next, the suitability of the various cell lines for tumor biology related studies was assessed. There are no standard models and selection criteria are not well defined. The activation of the Wnt pathway is a hallmark in colon carcinogenesis. Recent findings indicate that the Wnt activity defines CRC stem cells and is regulated by the microenvironment [28]. Thus, the overall Wnt signaling activity was compared in the various samples.
When comparing the average expression of direct Wnt targets (i.e. 24 Wnt target genes relevant to human colon and/or other cancers, with a proven direct transcriptional control through TCF binding sites as described in [29]), the SW620 cell line showed the strongest Wnt signal followed by SW480, laser-dissected tumor cells, COLO320, DLD1 and undifferentiated Caco-2 cells (Table 1, detailed description of all gene sets see supplementary data section "expression data analysis" and Additional file 1: Table S2). The ranking of cell lines with strong, medium and low average expression of

Table 1 Rank order of cell lines according to their signature strength

\begin{tabular}{|c|c|c|c|c|c|c|c|c|c|c|}
\hline Cell Line & EMT & HulSC & WNT & Trans_BC & SLC_SI3 & SLC_PM & $A B C$ & MKI67_CO & IC50_pos & IC50_neg \\
\hline$\overline{C A F}$ & 1 & 1 & 8 & 29 & 24 & 23 & 30 & 24 & 6 & 7 \\
\hline MB231 & 2 & 2 & 23 & 30 & 30 & 29 & 27 & 10 & 10 & 21 \\
\hline COLO320 & 3 & 11 & 4 & 17 & 27 & 28 & 10 & 4 & 4 & 29 \\
\hline SW480 & 4 & 4 & 2 & 28 & 25 & 27 & 17 & 17 & 24 & 12 \\
\hline SW620 & 5 & 9 & 1 & 27 & 29 & 22 & 20 & 8 & 7 & 26 \\
\hline Tumor & 6 & 3 & 3 & 20 & 8 & 24 & 26 & 23 & 5 & 20 \\
\hline Normal & 7 & 10 & 26 & 12 & 7 & 14 & 9 & 27 & 2 & 18 \\
\hline Caco,Fl,D & 8 & 15 & 14 & 6 & 6 & 5 & 6 & 21 & 8 & 13 \\
\hline Caco,Fl,U & 9 & 7 & 6 & 5 & 11 & 6 & 2 & 13 & 16 & 1 \\
\hline Caco_CDX2,U & 10 & 6 & 21 & 7 & 13 & 7 & 7 & 18 & 11 & 23 \\
\hline Caco_CDX2,D & 11 & 25 & 25 & 3 & 3 & 3 & 4 & 28 & 29 & 14 \\
\hline JE & 12 & 29 & 30 & 13 & 4 & 12 & 22 & 29 & 3 & 30 \\
\hline KM12 & 13 & 22 & 20 & 10 & 23 & 11 & 21 & 2 & 13 & 5 \\
\hline HCT116 & 14 & 12 & 24 & 21 & 26 & 25 & 18 & 14 & 9 & 24 \\
\hline Caco,Fi,D & 15 & 26 & 27 & 2 & 2 & 2 & 5 & 26 & 17 & 19 \\
\hline T84 & 16 & 17 & 18 & 26 & 9 & 17 & 29 & 22 & 22 & 25 \\
\hline CacoReady & 17 & 19 & 16 & 4 & 5 & 4 & 1 & 20 & 15 & 10 \\
\hline HT29,R & 18 & 16 & 11 & 24 & 16 & 10 & 13 & 5 & 25 & 3 \\
\hline DLD1 & 19 & 13 & 5 & 9 & 18 & 15 & 24 & 3 & 18 & 2 \\
\hline $\mathrm{Caco}, \mathrm{S}$ & 20 & 27 & 15 & 23 & 22 & 20 & 11 & 9 & 28 & 6 \\
\hline HT29,S & 21 & 18 & 13 & 18 & 15 & 13 & 12 & 1 & 20 & 4 \\
\hline MCF7 & 22 & 24 & 28 & 25 & 28 & 30 & 25 & 15 & 14 & 28 \\
\hline HT29,D & 23 & 28 & 22 & 22 & 10 & 21 & 16 & 25 & 26 & 8 \\
\hline Caco,R & 24 & 14 & 12 & 16 & 19 & 18 & 8 & 7 & 27 & 11 \\
\hline IL & 25 & 30 & 29 & 1 & 1 & 1 & 3 & 30 & 1 & 27 \\
\hline HT29,U & 26 & 23 & 19 & 19 & 14 & 9 & 23 & 16 & 30 & 17 \\
\hline LOVO & 27 & 5 & 7 & 14 & 21 & 16 & 19 & 6 & 12 & 15 \\
\hline LS174T & 28 & 8 & 10 & 15 & 12 & 26 & 28 & 19 & 23 & 16 \\
\hline COLO205 & 29 & 20 & 9 & 11 & 17 & 8 & 15 & 12 & 21 & 22 \\
\hline HCT15 & 30 & 21 & 17 & 8 & 20 & 19 & 14 & 11 & 19 & 9 \\
\hline
\end{tabular}

EMT = EMT-related genes, HulSC=“Humanized intestinal stem cell signature”, WNT = direct Wnt targets, Trans_BC = Transporters known to play important role in oral drug absorption, SLC_SI3 = solute carriers expressed at a significantly higher level in small intestinal samples versus colon samples, SLC_PM = solute carriers of the plasma membrane, $A B C=A B C$ transporters, MKI67_CO: Set of genes correlating with MKI67 expression in colon cancer samples, IC50_pos, IC50_neg: Genes with either strong positive or negative correlation between $\mathrm{GI} 50$ values of chemotherapeutics and expression across 60 cell line panel (for details of signatures see Additional file 6, methods). Tumor = laser dissected tumor cells, Normal = laser dissected colonocytes, JE, IL = laser dissected enterocytes of the jejunum or ileum, respectively, $\mathrm{D}=$ differentiated, $\mathrm{U}=$ undifferentiated, $\mathrm{Fi}=$ grown on filters, $\mathrm{FI}=$ grown in flasks, $\mathrm{R}=$ resistant to methotraxate, $\mathrm{S}=$ sensitive to methotrexate. 
direct Wnt targets was in agreement with the Wnt activity based on TOPFLASH assays reported by RosinArbesfeld and co-workers [4]. Moreover, the expression of genes involved in EMT ([29]) and a set of genes, recently being described as "humanized intestinal stem cell signature" (HuISC), which identified recurrent CRC [30], were studied to rank the cell lines as models for tumor cells at the invasive front and/or tumor cells with stemness characteristics. Survival analysis studies showed that risk of recurrence was significantly higher for CRC patients with a strong EMT signature and also correlated with overall survival (Figure 2). As expected CAFs and MB231 (positive controls) had the strongest expression of the EMT signature followed by COLO320, SW480, SW620 and laser-dissected tumor epithelial cells, normal colonocytes and differentiated Caco- 2 cells. Though COLO320 cells ranked highest of all intestinal epithelial cells, they also had the highest expression of CDX2, a key player in the maturation of intestinal enterocytes [31]. When the cell lines were ranked to the overall expression of stem cell related genes (HuISC), the five top ranked samples were $\mathrm{CAF}>\mathrm{MB} 231>$ laser-dissected tumor cells $>$ SW480 $>$ LOVO (Table 1). In summary, with respect to the Wnt, EMT and HuISC signatures, SW480 proved the cell line with the strongest traits and a gene expression pattern most similar to that of microdissected tumor epithelium.

\section{Gain of malignant traits and loss of differentiation} signature were associated with migratory properties Invasive tumor cells acquire higher motility through EMT [9]. Thus, we elucidated to what extent genomic expression characteristics could be linked to migratory properties. In general, the analysis revealed that higher migratory propensity was associated with strong EMT traits and weak expression of a signature derived from normal small intestinal epithelium (i.e. SLC_SI3). Accordingly, SW480 and MB231 cells showed the highest migratory capacity (Figure 3).

\section{Transporter profile defines absorption model}

The model of choice to evaluate drug absorption are Caco-2 cells as they undergo in vitro spontaneous enterocytic differentiation by developing an apical brush

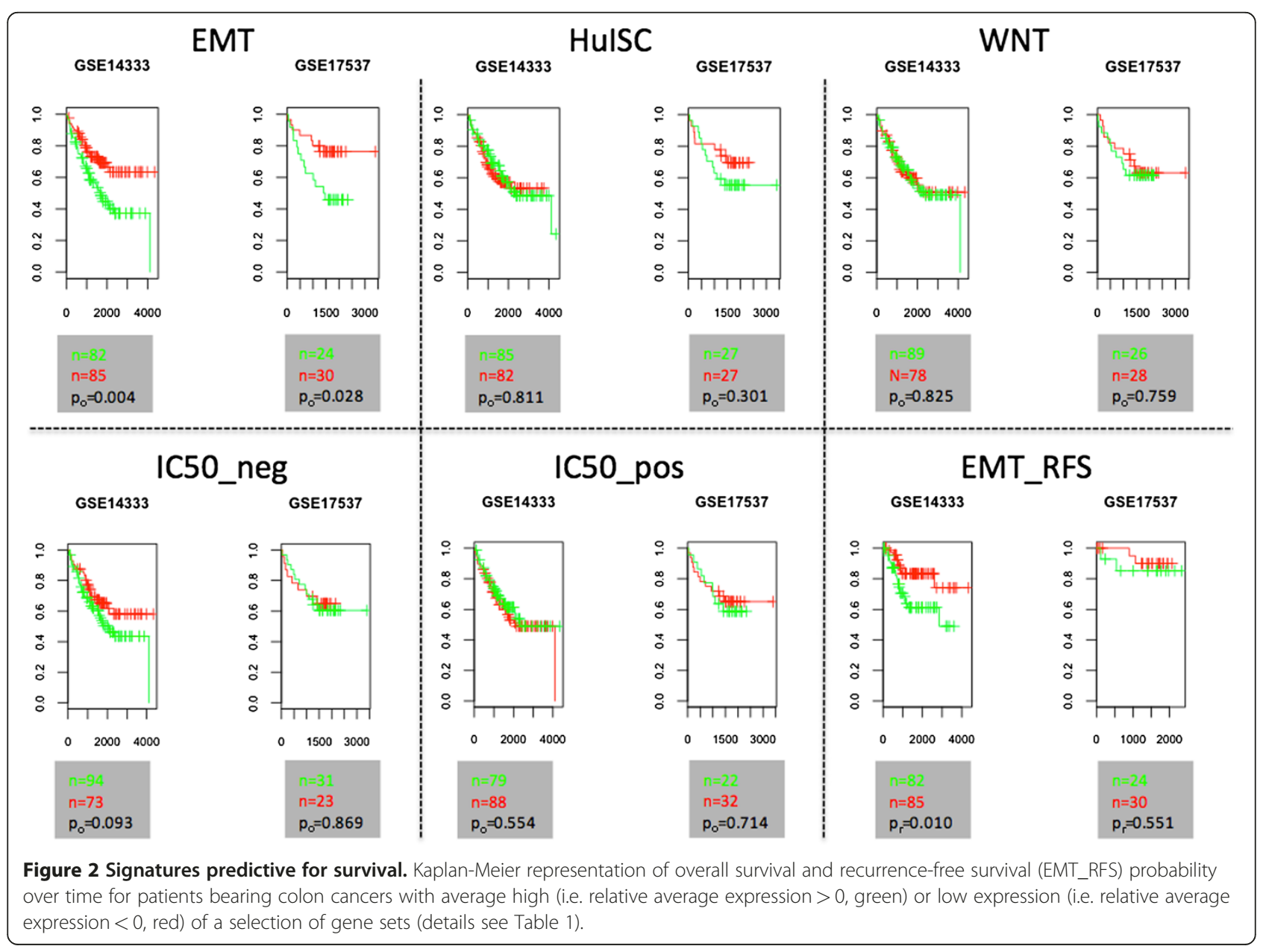




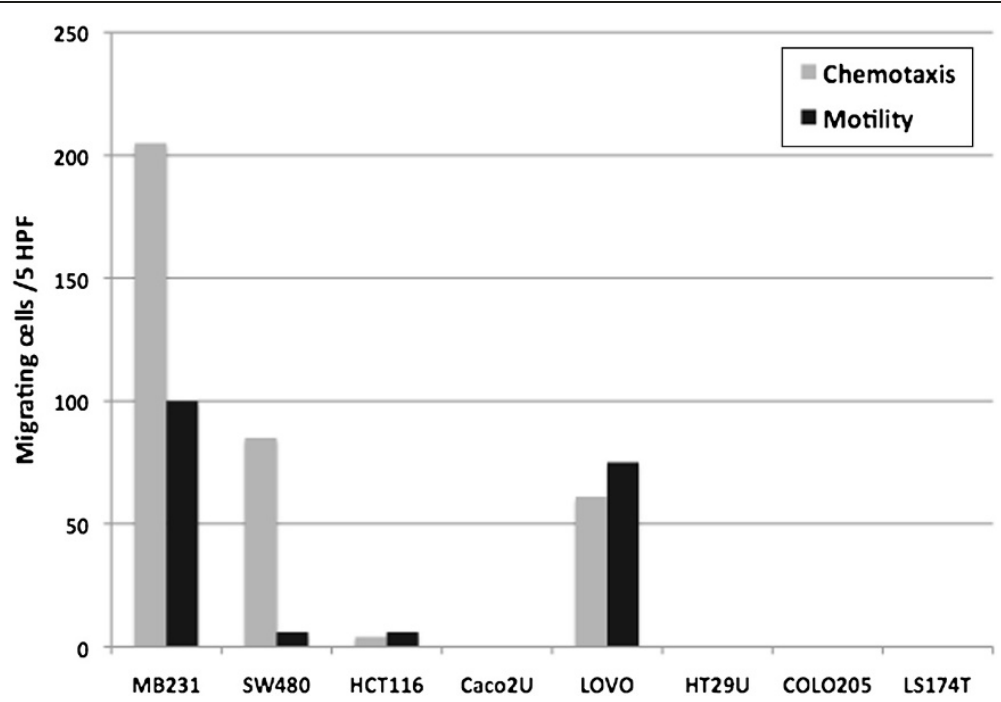

Figure 3 Gain of malignant traits and loss of differentiation signature are associated with migratory properties. Migratory properties of intestinal cell lines across membrane in the absence (motility) or presence (chemotaxis) of a FCS gradient (details see "Material and Methods" section). Cell lines are ordered in dependence of rank orders of EMT and SLC_SI3 signatures as shown in Table 1.

border membrane endowed with hydrolases such as sucrase-isomaltase and aminopeptidase $\mathrm{N}$ [7,32-36]. On the other hand, comparison of permeability coefficients across different laboratories is still an issue [37]. Various studies have compared the genomic profiles of Caco-2 cells to large and small intestinal tissue [5,38-40]. None of these has systematically compared these profiles to the ones of small intestinal enterocytes in comparison to other intestinal cell lines, and, in particular, to profiles of the genes relevant for active transport.

Consequently, we made use of our objective approach to assess the suitability of the Caco-2 cells as model for oral drug absorption as compared to other CRC derived cell lines, taking inter-laboratory differences into account. As described above, PCA suggested that T84, HT29 and Caco-2 cells grown to confluency for 3 weeks were most similar to small intestinal enterocytes. Yet, when performing PCA filtering on solute carriers, transporters mostly responsible for active uptake, differentiated Caco-2 cells emerged as most akin to small intestinal enterocytes (Figure 4). When the analysis was extended to include expression of drug transporters known to be involved in uptake and secretion in the small intestine (Trans_BC [41]), differentiated Caco-2 cells clustered with normal colonocytes as well as small intestinal enterocytes (Figure 5). However, when focusing only on transporters potentially relevant for uptake (i.e. solute carriers expressed in plasma membrane according to Gene Ontology, SLC_PM), differentiated Caco-2 cells clustered only with small intestinal enterocytes (Additional file 2: Figure $\mathrm{S} 1$ ). When focusing on $\mathrm{ABC}$ transporters, differentiated Caco-2 cells clustered with HT29 and T84 cells (Additional file 3: Figure S2), suggesting that these cell lines could also serve as a model for active export. Focusing on the expression of SLCs specific for the small intestine (i.e. SLC_SI3: SLCs expressed $\geq$ two fold in small intestine versus normal pool samples), differentiated Caco-2 cells, however, ranked highest independent of culture conditions and origins (Table 1).

\section{Selection criteria for chemo-sensitivity studies}

Recent reports suggest that chemotherapy may lead to a selection of tumor cells with EMT and stem cell properties and increased chemo-resistance [12]. For example, Gupta et al. [11] suggested that treatment with paclitaxel as compared to salinomycin significantly enriched breast

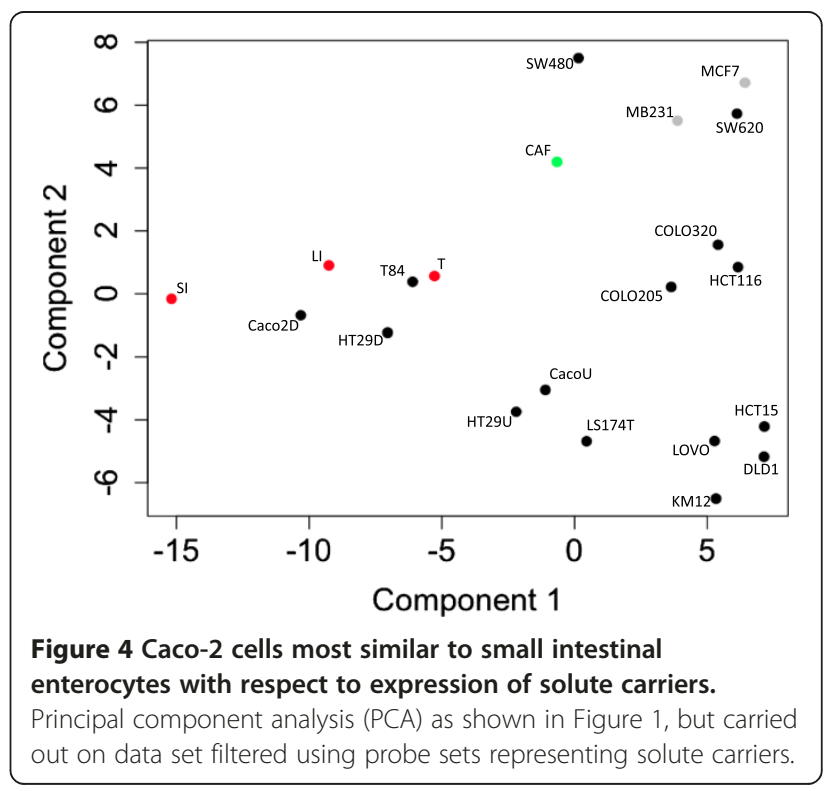




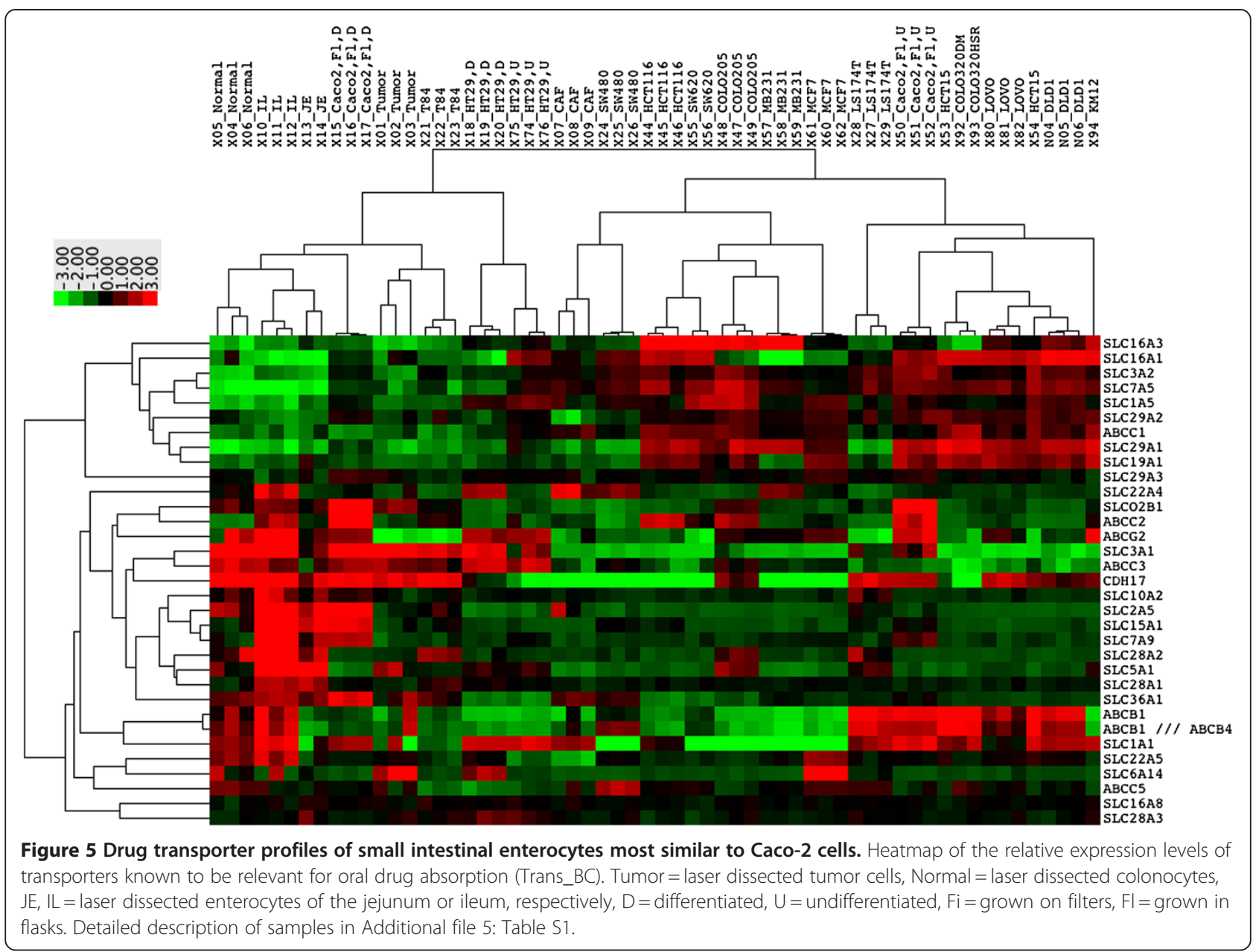

cancer cells with EMT properties. Thus, we examined their data set (GEO, GSE17215) and found that our EMT signature was also significantly enriched when performing GSEA $(p=0.000, F D R=0.000$, normalized enrichment score $=2.01$ ). Using the same approach, however, we could not observe any significant enrichment (i.e. $\mathrm{p}<0.05$ ) of this EMT signature in either oxaliplatin-treated HT29, DLD1 and LOVO cells (GEO, GSE10405), or methotrexate-treated HT29 and Caco-2 cells (GEO, GSE16648) as compared to untreated cells. Of note, however, that the stem cell signature HuISC was enriched in methotrexate treated Caco- 2 cells $(\mathrm{p}=0.000$, $\mathrm{FDR}=0.000$, normalized enrichment score $=2.08$ ).

To extend this analysis we addressed more comprehensively to what extent EMT, HuISC and WNT signatures correlated with GI50 values of 50 commonly used chemotherapeutics in the NCI60 cell line panel (http:// dtp.nci.nih.gov/docs/cancer/cancer_data.html).

Unexpectedly, no correlation between expression of EMT, HuISC and Wnt signatures and GI50 values was observed (Figure 6A), and furthermore, rank orders in terms of EMT, HuISC and Wnt signatures of 6 CRC cell lines comprised within the NCI60 panel (Table 1) did not reveal any positive correlation with GI50 values, yet even a tendency to a negative link (Figure 6B).

Most chemotherapeutics interfere with the cell cycle activities and accordingly cell proliferation has been associated with susceptibility to chemotherapy [42]. As expected, a positive correlation between expression of proliferation associated genes and GI50 values was observed across the NCI60 cell lines (Figure 6A). The CRC cell lines tested herein (Table 1) displayed no significant difference in proliferation (data not shown) and no link was observed between their rank orders (Table 1) and GI50 values (Figure 6B).

Finally, we scrutinized the NCI60 data sets for genes whose expression was either positively or negatively correlated with GI50 values of chemotherapeutics (i.e. for at least three compounds correlation coefficient $>|0.5|)$. Clustering of the correlation coefficients revealed two distinct gene sets (Additional file 4: Figure S3) for which the median positive and negative correlation coefficients were determined (Figure 6A). Interestingly, a link could be observed between rank orders of the CRC cell lines 

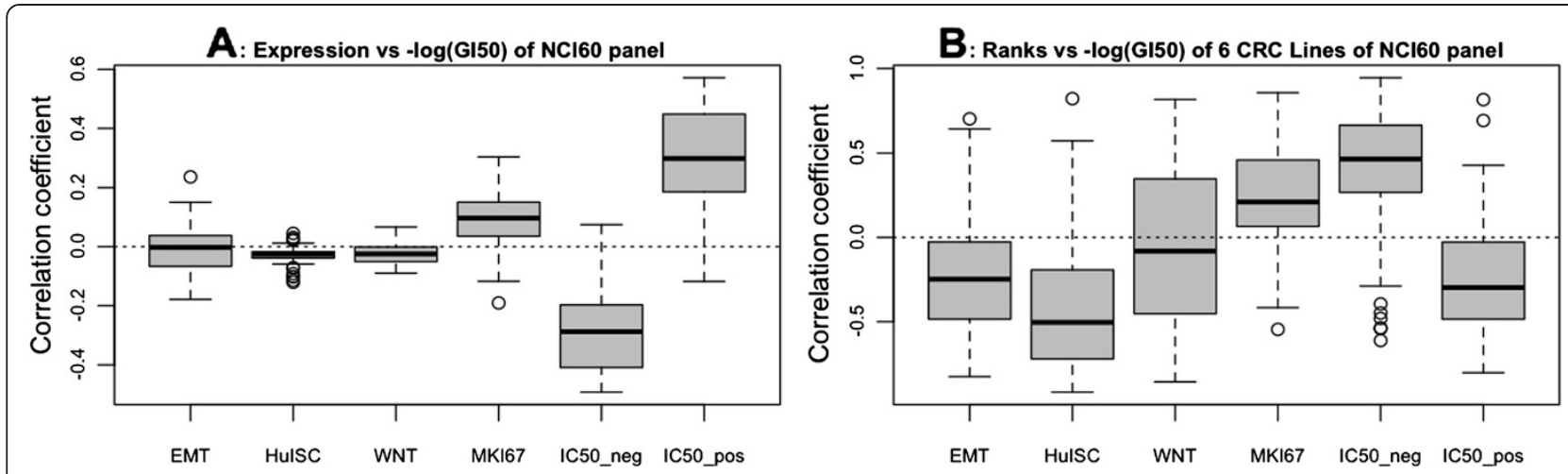

Figure 6 Expression of EMT and HuISC gene sets do not correlate with GI50 values, but with a signature defined by mining $\mathrm{NCI}$ databases. Boxplots of the median correlation coefficients between -log (Gl50) values and (A) expression of various gene sets in the NCl60 cell line panel or (B) rank orders according to Table 1 of the six intestinal cell lines represented in the NCI60 panel (i.e. SW620, HCT116, HCT15, COLO205, KM12, HT29). Gene sets as described in Table 1.

(Table 1) and GI50 values (Figure 6B), suggesting that these new signatures might be worth pursuing for assessing chemo-sensitivity.

\section{Discussion}

Selection criteria for cell-based models are often ill defined and arbitrary. Here we propose to make use of publicly available data generated by high throughput technologies to guide the choice of cell lines according to more objective criteria, such as gene expression signatures for selected traits. Focusing on CRC cell lines, we validated this approach for three different fields of application, i.e. the identification of cell lines most relevant for modeling and investigating tumor cell invasion, drug absorption and/or transport, and response to chemotherapy. Though we have focused on specific selection criteria, our strategy may be applied to any type of selection criteria (i.e. gene sets of different pathways and/or properties).

\section{Models to study tumor biology}

In view of the heterogeneity of solid tumors $[9,43]$ and the challenge to identify adequate cell-based models, we assessed to what extent comparative data mining for identifying tumor traits that overlap in tumor samples and cell lines might contribute to improving the relevance of cell-based models. We therefore ranked a panel of CRC cell lines using EMT and HuISC signatures to assess to what extent such signatures reflect invasive tumor behavior. Reports on in vivo studies with these cell lines indeed validate our ranking criteria. Thus, SW480, SW620, COLO320 and HCT116 cells, which all ranked high in our assessment for EMT and HuISC signatures, showed the highest propensity for local invasion upon orthotopic grafting [6,8], whereas HT29, COLO205 and DLD1, which ranked lower for these traits, showed less aggressive behavior. De Vries and coworkers observed that SW620 cells were indeed strongly invasive in vitro, in vivo, however, they proved less invasive than HT29 and LS174T cells [44]. Collectively, unbiased data mining allowed identifying cell lines with prominent mesenchymal traits (e.g. SW480), which display migratory properties and represent an invasive phenotype, whereas HT29, the most frequently used CRC model line, and T84 cells, which both ranked low for these traits came closest to gene expression profiles of bulk tumor tissue cells (Figure 1 and Table 1).

\section{Caco-2 cells, a model of choice for drug absorption}

Screening the literature with key words such as "absorption" and "transporters" revealed that, within the CRC cell line panel assessed herein, Caco-2 is the most widely used intestinal cell line for absorption-related studies [45-47]. Comparing transporter gene expression profiles revealed that differentiated Caco- 2 cells indeed most accurately recapitulate those of normal enterocytes. Similar to Hayeshi et al. [37], we observed that culture conditions influence the expression pattern of selected transporters. Based on our ranking method we show that Caco-2 cells grown for three weeks, independently of their origin and culture conditions, best match the genomic profiles of small intestinal enterocytes.

\section{Chemo-sensitivity}

Selection of tumor cells with EMT and stem cell properties and higher chemo-resistance has recently emerged as a novel mechanism underlying therapy-resistance $[10-12,48,49]$. Vast databases with GI50 values, a standard to assess drug sensitivity [15,16], are available for the intestinal CRC lines HT29, COLO205, KM12, SW620, HCT116, HCC_2998 and HCT15 through the NCI database (http://dtp.nci.nih.gov/docs/cancer/cancer_ 
data.html). We therefore probed these data sets for a correlation between EMT and CSC signatures and chemoresistance. Using the EMT signature defined herein, we could indeed confirm such a correlation in the breast cancer data set of Gupta et al. [11]. However, we could not observe any significant association of EMT-related genes with the GI50 values of CRC cell lines (Figure 6). Interestingly, novel chemoresistance-associated signatures could be identified; indicating that in CRC cell lines expression of EMT and CSC signatures are not dominant factors in the response to therapy. Cell line- or treatmentspecific effects may account for this discrepancy. It is of note that Gupta and co-authors compared paclitaxel vs. salinomycin-treated cells, while the other data were based on treatment versus non-treatment comparisons.

A correlation between drug resistance and EMT features has, however, been observed using CRC biopsies $[13,14]$. As these may contain variable amounts of stroma and as stroma content may influence response to therapy [50], these data are not directly comparable to GI50 values of CRC cell lines.

\section{Conclusions}

We have presented a straightforward strategy to exploit publicly available gene expression data to guide the choice of cell-based models. While this approach does not overcome the major limitations of such models, introducing a rank order of selected features may allow selecting model cell lines that are more adapted and pertinent to the addressed biological question. In summary, we conclude that SW480 represent an EMT-high, migratory phenotype and scored highest in terms of signatures associated to worse overall survival and higher risk of recurrence based on patient derived databases, therefore a good model to study invasive tumor cells. On the other hand, differentiated HT29 and T84 cells showed gene expression patterns closest to tumor bulk derived cells. Regarding drug absorption, we confirmed that differentiated Caco- 2 cells are the model of choice for active uptake studies in the small intestine.

\section{Methods}

\section{Cell culture}

Cell culture media were as follows: SW480, RPMI 1640, $10 \%$ fetal calf serum (FCS); Caco-2, Dulbecco's modified Eagle essential medium (DMEM) Glutamax, 10\% FCS, $1 \%$ non-essential amino acids; HT29, LS174T, DMEM Glutamax, 10\% FCS; T84, RPMI 1640, 10\% FCS, 2 mM glutamine. Primary fibroblasts, DMEM Glutamax, 10\% FCS, $100 \mathrm{U} / \mathrm{ml}$ penicillin, $100 \mu \mathrm{g} / \mathrm{ml}$ streptomycin, pt? $>0.5 \mathrm{~mL}$ gentamycin $(50 \mathrm{mg} / \mathrm{mL})$. All cell lines were cultured at $37^{\circ} \mathrm{C}$ and $5 \% \mathrm{CO}_{2}$ (Samples $15-29$ see Additional file 5: Table S1). CDX2-shCaco-2 cells were maintained under puromycin selection (2 ug/mL) (Samples 86-88 see
Additional file 5: Table S1). RNA was extracted using Nucleo-Spin RNA-extraction kit from Machery-Nagel (Oensingen, Switzerland). CacoReady ${ }^{\mathrm{TM}}$ kit cells (Readycell, Barcelona, Spain) were cultured on 24-transwells filters for either 14 days in DMEM low glucose containing $10 \% \mathrm{FCS}, 1 \% \mathrm{~L}$-glutamine and $100 \mathrm{U} / \mathrm{ml}$ penicillin, $100 \mu \mathrm{g} / \mathrm{ml}$ streptomycin, then 4 days of culture in semisolid shipping media and again 3 days in culture media (Readycell's patented technology) (Samples 54-56 see Additional file 5: Table S1), or for 3 weeks in the absence of the semi-solid shipping media (Samples 57-59 see Additional file 5: Table S1). Differentiated Caco-2LD cells were grown at low density and then differentiated on $0.4 \mu \mathrm{m}$ PET filters as described earlier [51] (Samples 49-51 see Additional file 5: Table S1).

\section{Laser dissection microscopy}

Immediately after resection of human tissue, samples were put on ice and sections of one cm length of small intestine and colon (tumor regions and adjacent normal epithelium), respectively, were cut, washed in chilled PBS, embedded in OCT and frozen immediately. One section of the ileum und two of the jejunum were first stored in RNAlater at $4{ }^{\circ} \mathrm{C}$ for shipping, then rinsed with chilled PBS and embedded in OCT and frozen immediately. $12 \mu \mathrm{m}$ frozen sections were cut and mounted on Leica or Zeiss membranes for dissecting microscopy (Leica Microsystems, Germany and PALM Microbeam, Germany), fixed in 96\% ethanol for $30 \mathrm{~s}$ and colored for an equivalent time with hematoxylin/eosin solution, respectively. Membranes were then rinsed in water for $30 \mathrm{~s}$, transferred for $10 \mathrm{~s}$ to $70 \%$ ethanol, followed by $96 \%$ ethanol and air-dried. Frozen samples were processed using a laser dissecting microscope coupled to a CCD camera (Leica Microsystems, Germany and PALM Microbeam, Germany). For each patient sample dissected cells were pooled in a tube cap containing $20 \mu \mathrm{L}$ RNA lysis buffer. Collection of human intestinal samples was approved by the corresponding local ethic commission (Samples 1-6, 10-14 see Additional file 5: Table S1).

\section{Gene expression using GeneChip ${ }^{\circledR}$ human genome U133} plus 2.0

Total RNA was extracted using the total RNA extraction Nucleospin II kit by Machery-Nagel (Oensingen, Switzerland). The quality and quantity of all RNA samples was examined by the Agilent 2100 Bioanalyzer (Agilent Biotechnologies, Germany) and by a NanoDrop (Witec AG, Switzerland), respectively. RINs for cell lines were between 9 and 10, for LDM material between 5 and 7 except for two small intestinal samples. $100 \mathrm{ng}$ of total RNA were used as the starting material for all individual samples. Labeling and fragmentation of cRNA, array hybridization and scanning was performed according to 
the protocol by Affymetrix. Details see Additional file 6 . Cell lines were measured in triplicates with different passage numbers. Normal tumor intestinal human tissue was obtained from different individuals. The complete data set is publicly available at http://www.ncbi.nlm.nih. gov/geo/ through the accession number GSE30292.

\section{Migration and proliferation assay}

Chemotaxis was assayed in 48-well Boyden microchambers (Neuro Probe, Cabin John, MD, USA). Cells were cultured in serum-free media overnight. For the migration studies, chemotaxis buffer containing 30\% serum was placed in the lower wells, and $10^{4}$ cells, suspended in chemotaxis buffer (culture media with 1\% BSA), in the upper wells. A PVPF membrane (Poretics $25 \times 80 \mathrm{~mm}$ ) with $8 \mu \mathrm{m}$ pores was coated with rat collagen type I. After $15 \mathrm{~h}$ of incubation the membrane was removed, washed on the upper side with PBS, fixed and stained with Diff-Quick staining kit. Migrated cells were counted at 1000-fold magnification in five randomly selected fields. Values are given as average cell count of five highpowered fields (5 HPF). Proliferation was assessed using an MTT assay (Sigma-Aldrich, Switzerland) over five days. Briefly, 3000 cells were seeded in five replicates per day, per well in a 96-well plate. At each time point $10 \mu \mathrm{L}$ MTT working solution was added to each well. Then the plate was incubated for $3 \mathrm{~h}$ at $37^{\circ} \mathrm{C}$ and the cells lysed with lysis buffer overnight. Optical density was measured at $540 \mathrm{~nm}$. ANOVA was done for migration data ( $\mathrm{p}$ value $\leq 0.05$ ).

\section{Public data}

Publicly available data was obtained at http://www.ncbi. nlm.nih.gov/geo/ through the accession numbers GSE2361, GSE2361, GSE7303 (Pool normal; colon GSM175905, small intestine GSM175908, lung GSM176012, breast GSM175792, prostate GSM175923, uterus GSM175945, kidney GSM175911), GSE2109 (Pool tumor; colon GSM38055, small intestine GSM38068, lung GSM38051, breast GSM38051, prostate GSM38053, uterus GSM38052, kidney GSM38073), GSE10843, GSE13059, GSE16648, GSE22572. MB231 and MCF7 cell lines (GSE10890) were included in the analysis as controls for cells with strong EMT properties and with weak, respectively. Expression data for NCI60 cell line panel was obtained through http://discover.nci.nih.gov/cellminer/ [52] and GI50 data at http://dtp.nci.nih.gov/docs/cancer/ cancer_data.html.

\section{Expression data analysis}

Robust multi-array averaging (RMA) and quantile normalization were used to quantify gene expression. Significant differences were identified applying a Bayesian approach using the limma package ( $R$ 2.12.0,
Bioconductor 2.7). A threshold of an adjusted $\mathrm{p}$ value $\leq$ 0.05 was used to identify significant changes if not indicated otherwise. Gene set enrichment analysis (GSEA) was carried out according to Subramanian et al. [53] and $\mathrm{p}$ values were computed using a bootstrap distribution created by resampling gene sets of the same cardinality. See details in supplementary data section "expression data analysis" and Additional file 1: Table S2 regarding data sets. Principle component analysis was performed using the affy package ( $R$, Bioconductor). Correlation coefficients between $-\log ($ GI50) of selected drugs (see Additional file 6) and normalized expression values were calculated using R. Heatmaps were generated using Cluster 3.0 and TreeView (http://rana.lbl.gov/EisenSoftware. htm) [54]. Centered correlation or Spearman rank correlation and average linkage clustering, respectively, were used for similarity measurement and clustering. Effects of signatures on overall and recurrence free survival were studied using the Kaplan-Meier survival curves and log rank tests in three different publicly available data sets (GSE12945, GSE14333, GSE17537) using the survival package in the statistical software $\mathrm{R}$ version 2.13.0. Patient samples were divided into two groups splitting at the average score.

\section{Ranking according to signature strength}

Gene expression data was filtered by selecting only one probe set, the one with the highest standard deviation across all samples, per gene. For each gene of a given gene set the relative expression (i.e. gene-wise zerocentering of expression values) across all samples was determined. The score of a gene set was calculated as the average of the expression values of the genes in the set. The cell lines were then ranked according to this score. See details in supplementary data section "expression data analysis" and Additional file 1: Table S2 regarding data sets.

\section{Drug sensitivity analysis}

Correlation coefficients between $-\log ($ GI50) and $\log 2$ gene expression values were calculated for a panel of 50 chemotherapeutics (CTX, list of drugs see Additional file 7: Figure S4) and a panel of drugs (NSC) for which for all 59 cell lines GI50 values were available and SD across 59 lines of $-\log (\mathrm{GI} 50)$ was $>0$.

\section{Additional files}

Additional file 1: Table S2. Gene sets used for data analysis.

Additional file 2: Figure S1. Solute carriers of plasma membrane profiles of small intestinal enterocytes most similar to Caco- 2 cells. Heatmap of relative expression levels of solute carriers of the plasma membrane. 
Additional file 3: Figure S2. Differentiated Caco-2, HT29 and T84 cell lines most similar to enterocytes with respect to expression $A B C$ transporters. Heatmap of the relative expression levels of $A B C$ transporters.

Additional file 4: Figure S3. Two distinct groups of genes with respect to expected chemosensitivity. Heatmap of correlation coefficients $-\log (\mathrm{GI} 50)$ of 50 chemotherapeutics and expression values across the $\mathrm{NCl} 60$ cell line panel.

Additional file 5: Table 1S. List of expression files used for microarray analysis and corresponding accession numbers at Gene Expression Omnibus.

Additional file 6: Supplementary information for methods and additional results $[29,30,41,55-58]$.

Additional file 7: Figure S4. Enrichment of small intestinal signature in Caco-2 cells upon differentiation. Heatmap representing enrichment scores obtained by gene set enrichment analysis of the selected panel of gene sets. Genes in the various cell lines were ranked according to their relative average expression $(n=2-3)$ in the given cell line compared to a panel of healthy epithelial tissues. Details see Additional file 6, material and methods.

\section{Competing interests}

The authors declare that they have no competing interests.

\section{Acknowledgments}

The ileum samples used for LDM were kindly provided by Jose Castell (University Hospital 'La Fe', Valencia, Spain) and Biopredic International (Rennes, France). Microarray hybridization was performed by Genomic Technologies Facility of Center for Integrative Genomics, (University of Lausanne, Lausanne, Switzerland) and Genomics Platform NCCR "Frontiers in Genetics" (University of Geneva, Geneva, Switzerland). We also would like to thank Cristina Bernadó (Vall d'Hebron Institute of Oncology (VHIO, Barcelona, Spain) and Myriam Fabre (Advancell, Barcelona, Spain) for or technical support and helpful discussions. This research was supported by Oncosuisse through project OC-02302-08-2008, the 6th Framework programme, LSH-2005-1.2.3-4 through project Liintop (037499), the Swiss National Science Foundation (SNSF) through the National Center of Competence in Research (NCCR) TransCure and Fondazione San Salvatore.

\section{Author details}

'Institute for Macromolecular Chemistry and Center for Biological Signaling Studies (BIOSS), University of Freiburg, Freiburg, Germany. ${ }^{2}$ Institute of Biochemistry and Molecular Medicine, University of Berne, Berne, Switzerland. ${ }^{3}$ Istituto di Biologia Cellulare e Neurobiologia, CNR, Rome, Italy. ${ }^{4}$ OSC - Omics Science Center, RIKEN Yokohama Institute, Yokohama, Japan. ${ }^{5}$ Swiss Institute of Bioinformatics, Lausanne, Switzerland. 'S ${ }^{6}$ wiss National Centre of Competence in Research Molecular Oncology, Swiss Institute for Experimental Cancer Research, Ecole Polytechnique Fédérale de Lausanne, School of Life Sciences, Lausanne, Switzerland. ${ }^{7}$ NCCR TransCure, University of Berne, Berne, Switzerland. ${ }^{8}$ Institute of pathology of Lausanne, Centre hospitalier et universitaire vaudois, Lausanne, Switzerland. ' ${ }^{\circ}$ Laboratorio de Investigaciones del Sistema Inmune (LISIN), Facultad de Ciencias Exactas, Universidad Nacional de La Plata, La Plata, Argentina. ${ }^{10}$ Faculty of Pharmacy, University of Helsinki, Helsinki, Finland. "1"LEITAT Technological Center, Barcelona, Spain. ${ }^{12}$ Medical Oncology Department, IOSI, Ospedale Regionale di Lugano, Lugano, Switzerland. ${ }^{13}$ Swiss Institute for Experimental Cancer Research, Ecole Polytechnique Fédérale de Lausanne, School of Life Sciences, Lausanne, Switzerland. ${ }^{14}$ Institute of Biochemistry and Molecular Medicine, University of Bern, Buehlstrasse 28, 3000, Bern 9, Switzerland.

\section{Authors' contributions}

Data acquisition: JC, SE, MN, PA, SB, MV, AF, SS, JH; data analysis: PA, JC, SE, TS, MD, MA; patient samples: MR, HB, PS, JC, PA; study design, funding, drafting of ms: PA, MA; supervision: PA. All authors read and approved the final manuscript.

Received: 13 April 2012 Accepted: 22 June 2012

Published: 22 June 2012

\section{References}

1. Markowitz SD, Bertagnolli MM: Molecular origins of cancer: Molecular basis of colorectal cancer. N Engl J Med 2009, 361(25):2449-2460.

2. Gayet J, Zhou XP, Duval A, Rolland S, Hoang JM, Cottu P, Hamelin R: Extensive characterization of genetic alterations in a series of human colorectal cancer cell lines. Oncogene 2001, 20(36):5025-5032.

3. Oliveira C, Pinto M, Duval A, Brennetot C, Domingo E, Espin E, Armengol M, Yamamoto $\mathrm{H}$, Hamelin $\mathrm{R}$, Seruca $\mathrm{R}$, et al: BRAF mutations characterize colon but not gastric cancer with mismatch repair deficiency. Oncogene 2003, 22(57):9192-9196.

4. Rosin-Arbesfeld R, Cliffe A, Brabletz T, Bienz M: Nuclear export of the APC tumour suppressor controls beta-catenin function in transcription. EMBO J 2003, 22(5):1101-1113.

5. van Erk MJ, Krul CA, Caldenhoven E, Stierum RH, Peters WH, Woutersen RA, van Ommen B: Expression profiling of colon cancer cell lines and colon biopsies: towards a screening system for potential cancer-preventive compounds. Eur J Cancer Prev 2005, 14(5):439-457.

6. Cespedes MV, Espina C, Garcia-Cabezas MA, Trias M, Boluda A, Gomez del Pulgar MT, Sancho FJ, Nistal M, Lacal JC, Mangues R: Orthotopic microinjection of human colon cancer cells in nude mice induces tumor foci in all clinically relevant metastatic sites. Am J Pathol 2007, 170(3):1077-1085.

7. Chantret I, Barbat A, Dussaulx E, Brattain MG, Zweibaum A: Epithelial polarity, villin expression, and enterocytic differentiation of cultured human colon carcinoma cells: a survey of twenty cell lines. Cancer Res 1988, 48(7):1936-1942.

8. Flatmark K, Maelandsmo GM, Martinsen M, Rasmussen H, Fodstad O: Twelve colorectal cancer cell lines exhibit highly variable growth and metastatic capacities in an orthotopic model in nude mice. Eur J Cancer 2004, 40(10):1593-1598.

9. Thiery JP, Sleeman JP: Complex networks orchestrate epithelialmesenchymal transitions. Nat Rev Mol Cell Biol 2006, 7(2):131-142.

10. Mani SA, Guo W, Liao MJ, Eaton EN, Ayyanan A, Zhou AY, Brooks M, Reinhard F, Zhang CC, Shipitsin M, et al: The epithelial-mesenchymal transition generates cells with properties of stem cells. Cell 2008, 133(4):704-715.

11. Gupta PB, Onder TT, Jiang G, Tao K, Kuperwasser C, Weinberg RA, Lander ES: Identification of selective inhibitors of cancer stem cells by high-throughput screening. Cell 2009, 138(4):645-659.

12. Singh A, Settleman J: EMT, cancer stem cells and drug resistance: an emerging axis of evil in the war on cancer. Oncogene 2010, 29(34):4741-4751.

13. Loboda A, Nebozhyn MV, Watters JW, Buser CA, Shaw PM, Huang PS, Van't Veer L, Tollenaar RA, Jackson DB, Agrawal D, et al: EMT is the dominant program in human colon cancer. BMC Med Genet 2011, 4:9.

14. Oh SC, Park YY, Park ES, Lim JY, Kim SM, Kim SB, Kim J, Kim SC, Chu IS, Smith JJ, et al: Prognostic gene expression signature associated with two molecularly distinct subtypes of colorectal cancer. Gut 2011.

15. Sharma SV, Haber DA, Settleman J: Cell line-based platforms to evaluate the therapeutic efficacy of candidate anticancer agents. Nat Rev Cancer 2010, 10(4):241-253.

16. Shoemaker $\mathrm{RH}$ : The $\mathrm{NCl} 60$ human tumour cell line anticancer drug screen. Nat Rev Cancer 2006, 6(10):813-823.

17. Scherf U, Ross DT, Waltham M, Smith LH, Lee JK, Tanabe L, Kohn KW, Reinhold WC, Myers TG, Andrews DT, et al: A gene expression database for the molecular pharmacology of cancer. Nat Genet 2000, 24(3):236-244.

18. Arango D, Mariadason JM, Wilson AJ, Yang W, Corner GA, Nicholas C, Aranes MJ, Augenlicht LH: c-Myc overexpression sensitises colon cancer cells to camptothecin-induced apoptosis. Br J Cancer 2003, 89(9):1757-1765.

19. Augenlicht $L H$, Velcich A, Klampfer L, Huang J, Corner G, Aranes M, Laboisse C, Rigas B, Lipkin M, Yang K, et al: Application of gene expression profiling to colon cell maturation, transformation and chemoprevention. J Nutr 2003, 133(7 Suppl):2410S-2416S.

20. Boyer J, Allen WL, McLean EG, Wilson PM, McCulla A, Moore S, Longley DB, Caldas C, Johnston PG: Pharmacogenomic identification of novel determinants of response to chemotherapy in colon cancer. Cancer Res 2006, 66(5):2765-2777.

21. Hoshino H, Miyoshi N, Nagai K, Tomimaru $Y$, Nagano H, Sekimoto M, Doki $Y$, Mori M, Ishii H: Epithelial-mesenchymal transition with expression of 
SNAl1-induced chemoresistance in colorectal cancer. Biochem Biophys Res Commun 2009, 390(3):1061-1065.

22. Jung JJ, Jeung HC, Lee JO, Kim TS, Chung HC, Rha SY: Putative chemosensitivity predictive genes in colorectal cancer cell lines for anticancer agents. Oncol Rep 2007, 18(3):593-599.

23. Mariadason JM, Arango D, Augenlicht LH: Customizing chemotherapy for colon cancer: the potential of gene expression profiling. Drug Resist Updat 2004, 7(3):209-218.

24. Sabbah M, Emami S, Redeuilh G, Julien S, Prevost G, Zimber A, Ouelaa R, Bracke M, De Wever O, Gespach C: Molecular signature and therapeutic perspective of the epithelial-to-mesenchymal transitions in epithelial cancers. Drug Resist Updat 2008, 11(4-5):123-151.

25. Spitzner M, Emons G, Kramer F, Gaedcke J, Rave-Frank M, Scharf JG, Burfeind $P$, Becker $H$, Beissbarth T, Ghadimi BM, et al: A gene expression signature for chemoradiosensitivity of colorectal cancer cells. Int J Radiat Oncol Biol Phys 2010, 78(4):1184-1192

26. Staunton JE, Slonim DK, Coller HA, Tamayo P, Angelo MJ, Park J, Scherf U, Lee JK, Reinhold WO, Weinstein JN, et al: Chemosensitivity prediction by transcriptional profiling. Proc Natl Acad Sci U S A 2001, 98(19):10787-10792.

27. Neve RM, Chin K, Fridlyand J, Yeh J, Baehner FL, Fevr T, Clark L, Bayani N, Coppe JP, Tong F, et al: A collection of breast cancer cell lines for the study of functionally distinct cancer subtypes. Cancer Cell 2006, 10(6):515-527

28. Vermeulen L, De Sousa EMF, van der Heijden M, Cameron K, de Jong JH, Borovski T, Tuynman JB, Todaro M, Merz C, Rodermond H, et al: Wnt activity defines colon cancer stem cells and is regulated by the microenvironment. Nat Cell Biol 2010, 12(5):468-476.

29. Deka J, Wiedemann N, Anderle P, Murphy-Seiler F, Bultinck J, Eyckerman S, Stehle JC, Andre S, Vilain N, Zilian O, et al: Bcl9/Bcl9l are critical for Wnt-mediated regulation of stem cell traits in colon epithelium and adenocarcinomas. Cancer Res 2010, 70(16):6619-6628.

30. Merlos-Suarez A, Barriga FM, Jung P, Iglesias M, Cespedes MV, Rossell D, Sevillano M, Hernando-Momblona X, da Silva-Diz V, Munoz P: The Intestinal Stem Cell Signature Identifies Colorectal Cancer Stem Cells and Predicts Disease Relapse. Cell Stem Cell 2011, 8(5):511-524.

31. Guo RJ, Suh ER, Lynch JP: The role of Cdx proteins in intestinal development and cancer. Cancer Biol Ther 2004, 3(7):593-601.

32. Artursson P, Palm K, Luthman K: Caco-2 monolayers in experimental and theoretical predictions of drug transport. Adv Drug Deliv Rev 2001, 46(1-3):27-43

33. Fleet JC, Wang L, Vitek O, Craig BA, Edenberg HJ: Gene expression profiling of Caco-2 BBe cells suggests a role for specific signaling pathways during intestinal differentiation. Physiol Genomics 2003, 13(1):57-68.

34. Mariadason JM, Bordonaro M, Aslam F, Shi L, Kuraguchi M, Velcich A Augenlicht LH: Down-regulation of beta-catenin TCF signaling is linked to colonic epithelial cell differentiation. Cancer Res 2001, 61(8):3465-3471.

35. Pshezhetsky AV, Fedjaev M, Ashmarina L, Mazur A, Budman L, Sinnett D, Labuda D, Beaulieu JF, Menard D, Nifant'ev I, et al: Subcellular proteomics of cell differentiation: quantitative analysis of the plasma membrane proteome of Caco-2 cells. Proteomics 2007, 7(13):2201-2215.

36. Saaf AM, Halbleib JM, Chen X, Yuen ST, Leung SY, Nelson WJ, Brown PO: Parallels between global transcriptional programs of polarizing Caco-2 intestinal epithelial cells in vitro and gene expression programs in normal colon and colon cancer. Mol Biol Cell 2007, 18(11):4245-4260.

37. Hayeshi R, Hilgendorf C, Artursson P, Augustijns P, Brodin B, Dehertogh P, Fisher K, Fossati L, Hovenkamp E, Korjamo T, et al: Comparison of drug transporter gene expression and functionality in Caco-2 cells from 10 different laboratories. Eur J Pharm Sci 2008, 35(5):383-396.

38. Anderle P, Rakhmanova V, Woodford K, Zerangue N, Sadee W: Messenger RNA expression of transporter and ion channel genes in undifferentiated and differentiated Caco-2 cells compared to human intestines. Pharm Res 2003, 20(1):3-15

39. Calcagno AM, Ludwig JA, Fostel JM, Gottesman MM, Ambudkar SV: Comparison of drug transporter levels in normal colon, colon cancer, and Caco-2 cells: impact on drug disposition and discovery. Mol Pharm 2006, 3(1):87-93.

40. Tremblay E, Auclair J, Delvin E, Levy E, Menard D, Pshezhetsky AV, Rivard N, Seidman EG, Sinnett D, Vachon PH, et al: Gene expression profiles of normal proliferating and differentiating human intestinal epithelial cells: a comparison with the Caco-2 cell model. J Cell Biochem 2006 99(4):1175-1186.
41. Anderle P, Nielsen CU: Transporters in the gastrointestinal tract. In Drug Bioavailability: The Future of Integrative Metabolism. Edited by van de Waterbeemd H, Testa B.: Wiley VCH; 2008.

42. Mellor HR, Callaghan R: Resistance to chemotherapy in cancer: a complex and integrated cellular response. Pharmacology 2008, 81(4):275-300.

43. Hanahan D, Weinberg RA: Hallmarks of cancer: the next generation. Cell 2011, 144(5):646-674.

44. de Vries JE, Dinjens WN, De Bruyne GK, Verspaget HW, van der Linden EP, de Bruine AP, Mareel MM, Bosman FT, ten Kate J: In vivo and in vitro invasion in relation to phenotypic characteristics of human colorectal carcinoma cells. Br J Cancer 1995, 71(2):271-277.

45. Press B, Di Grandi D: Permeability for intestinal absorption: Caco-2 assay and related issues. Curr Drug Metab 2008, 9(9):893-900.

46. Sambuy Y, De Angelis I, Ranaldi G, Scarino ML, Stammati A, Zucco F: The Caco-2 cell line as a model of the intestinal barrier: influence of cell and culture-related factors on Caco-2 cell functional characteristics. Cell Biol Toxicol 2005, 21(1):1-26.

47. Sun H, Chow EC, Liu S, Du Y, Pang KS: The Caco-2 cell monolayer: usefulness and limitations. Expert Opin Drug Metab Toxicol 2008, 4(4):395-411.

48. Alison MR, Lim SM, Nicholson L: Cancer stem cells: problems for therapy? J Pathol 2011, 223(2):147-161

49. Zhou BB, Zhang H, Damelin M, Geles KG, Grindley JC, Dirks PB: Tumour-initiating cells: challenges and opportunities for anticancer drug discovery. Nat Rev Drug Discov 2009, 8(10):806-823.

50. Farmer $P$, Bonnefoi $H$, Anderle $P$, Cameron $D$, Wirapati $P$, Becette $V$, Andre $S$, Piccart M, Campone M, Brain E, et al: A stroma-related gene signature predicts resistance to neoadjuvant chemotherapy in breast cancer. Nat Med 2009, 15(1):68-74.

51. Natoli M, Leoni BD, D'Agnano I, D'Onofrio M, Brandi R, Arisi I, Zucco F, Felsani A: Cell growing density affects the structural and functional properties of Caco-2 differentiated monolayer. J Cell Physiol 2011, 226(6):1531-1543.

52. Pfister TD, Reinhold WC, Agama K, Gupta S, Khin SA, Kinders RJ, Parchment RE, Tomaszewski JE, Doroshow JH, Pommier Y: Topoisomerase I levels in the $\mathrm{NCl}-60$ cancer cell line panel determined by validated ELISA and microarray analysis and correlation with indenoisoquinoline sensitivity. Mol Cancer Ther 2009, 8(7):1878-1884.

53. Subramanian A, Tamayo P, Mootha VK, Mukherjee S, Ebert BL, Gillette MA, Paulovich A, Pomeroy SL, Golub TR, Lander ES, et al: Gene set enrichment analysis: a knowledge-based approach for interpreting genome-wide expression profiles. Proc Natl Acad Sci U S A 2005, 102(43):15545-15550.

54. Eisen MB, Spellman PT, Brown PO, Botstein D: Cluster analysis and display of genome-wide expression patterns. Proc Natl Acad Sci U S A 1998, 95(25):14863-14868.

55. Verzi MP, Shin H, He HH, Sulahian R, Meyer CA, Montgomery RK, Fleet JC, Brown M, Liu XS, Shivdasani RA: Differentiation-specific histone modifications reveal dynamic chromatin interactions and partners for the intestinal transcription factor CDX2. Dev Cell 2010, 19(5):713-726.

56. Gassler N, Newrzella D, Bohm C, Lyer S, Li L, Sorgenfrei O, van Laer L, Sido B, Mollenhauer J, Poustka A, et al: Molecular characterisation of nonabsorptive and absorptive enterocytes in human small intestine. Gut 2006, 55(8):1084-1089.

57. van der Flier LG, van Gijn ME, Hatzis P, Kujala P, Haegebarth A, Stange DE, Begthel $H$, van den Born M, Guryev $V$, Oving I, et al: Transcription factor achaete scute-like 2 controls intestinal stem cell fate. Cell 2009, 136 (5):903-912

58. Fritzmann J, Morkel M, Besser D, Budczies J, Kosel F, Brembeck FH, Stein U, Fichtner I, Schlag PM, Birchmeier W: A colorectal cancer expression profile that includes transforming growth factor beta inhibitor BAMBI predicts metastatic potential. Gastroenterology 2009, 137(1):165-175.

doi:10.1186/1471-2164-13-274

Cite this article as: Christensen et al:: Defining new criteria for selection of cell-based intestinal models using publicly available databases. BMC Genomics 2012 13:274. 